\title{
A High-Fidelity Batch Simulation Environment for Integrated Batch and Piloted Air Combat Simulation Analysis
}

\author{
Kenneth H. Goodrich* and Dr. John W. McManus** \\ NASA Langley Research Center, Hampton Virginia \\ Alan R. Chappell ${ }^{\dagger}$ \\ Lockheed Engineering \& Sciences Company, Hampton Virginia
}

\begin{abstract}
A batch air combat simulation environment known as the Tactical Maneuvering Simulator (TMS) is presented. The TMS serves as a tool for developing and evaluating tactical maneuvering logics. The environment can also be used to evaluate the tactical implications of perturbations to aircraft performance or supporting systems. The TMS is capable of simulating air combat between any number of engagement participants, with practical limits imposed by computer memory and processing power. Aircraft are modeled using equations of motion, control laws, aerodynamics and propulsive characteristics equivalent to those used in high-fidelity piloted simulation. Databases representative of a modern high-performance aircraft with and without thrust-vectoring capability are included. To simplify the task of developing and implementing maneuvering logics in the TMS, an outer-loop control system known as the Tactical Autopilot (TA) is implemented in the aircraft simulation model. The TA converts guidance commands issued by computerized maneuvering logics in the form of desired angle-of-attack and wind axisbank angle into inputs to the inner-loop control augmentation system of the aircraft. This report describes the capabilities and operation of the TMS.
\end{abstract}

\section{Introduction}

As new technologies or capabilities are proposed for inclusion in high-performance aircraft, it is imperative to assess the impact, utilization, and costs of these technologies within the context of air combat tactics and effectiveness. Due to the highly complex

\footnotetext{
* Research Engineer, Member AIAA

** Aerospace Engineer

$\dagger \quad$ Senior Engineer, P.E., Member AIAA Copyright (C) 1992 by the AIAA, Inc. No copyright is asserted in the United States under Title 17, U.S. Code. The U.S. Government has a royalty-free license to exercise all rights under the copyright claimed herein for Governmental purposes. All other rights are reserved for the copyright owner.
}

and transient nature of air combat, simulation is the primary tool for performing this assessment. Both batch and real-time, piloted simulation can contribute to the assessment. Batch air combat simulations such as the Advanced Air-to-Air System Performance Model (AASPEM, Reference 1) and TAC BRAWLER (Reference 2) allow the study of aircraft tactics and performance in a highly controlled and repeatable environment. Batch air combat simulations consist of two fundamental elements--computerized maneuvering logics which generate maneuver decisions and a simulation environment in which maneuvering logics are developed and tested. These programs can run a large number of engagements with minimal operator intervention, allowing comprehensive sets of initial conditions or parametric variations to be rapidly evaluated. Unfortunately, the minimal operator intervention inherent in batch operation slows development and validation of new maneuvering logics, resulting in a relatively inflexible set of tactics which may not effectively exploit a given situation or aircraft capability. In contrast, piloted simulation provides an environment ideally suited for rapid tactical experimentation and adaptation. New tactics can be investigated by simply instructing pilots to maneuver in the desired manner. Furthermore, the natural interface provided to the pilots encourages their participation in this development process and enhances their ability to assess the success of a given tactic. Unfortunately, due to the variability introduced by human pilots, the length of time required to perform a statistically meaningful piloted air combat simulation study, combined with the availability and expense of the necessary facilities and pilots, makes a comprehensive study extremely difficult to perform.

Because the strengths and weaknesses of batch and piloted simulation are complimentary, a synergism exists when the two approaches are employed in concert.

To fully exploit this synergy, NASA Langley Research Center is developing an integrated batch and piloted simulation tool known as the Tactical Guidance Research and Evaluation System (TiGRES, Reference 
3). TiGRES consists of three primary elements: an advanced, real-time-capable, artificial intelligence-based maneuvering logic (Reference 4), a multi-dome, piloted simulation facility known as the Differential Maneuvering Simulator (DMS, Reference 5), and a batch simulation environment known as the Tactical Maneuvering Simulator (TMS). The development and operation of the TMS and its relation to the other elements of TiGRES are the focuses of this paper.

Unlike existing batch air combat simulation environments which typically use reduced order dynamic models, aircraft in the TMS are modeled using equations of motion, control laws, aerodynamics and propulsive characteristics identical to those used in high-fidelity piloted simulation in the DMS. This commonality allows maneuvering logics developed in the TMS to be evaluated, without modification, against human pilots in the DMS. The ability to test maneuvering logics against human pilots provides an efficient means of validating the results of batch simulation analysis. Thus, extensive preliminary investigations of tactical maneuvering strategies, guidance concepts or aircraft performance characteristics can be performed quickly and cheaply using the TMS. After the focus of an investigation matures, a minimum amount of piloted simulation in the DMS can be used to confirm or refine the findings of the more comprehensive batch analysis.

The TMS can be broken into three basic elements. The first element is the simulation model used for simulating individual aircraft. Currently, models representative of a modern high-performance aircraft with and without thrust-vectoring capability are available. The second element is the Tactical Autopilot (TA). The TA is an autopilot which enables maneuvering logics to command full-order dynamic aircraft models in both the TMS and DMS. The TA converts guidance commands issued in the form of desired angle-of-attack and wind-axis bank angle into inputs to the inner-loop control augmentation system of the simulated aircraft. The final element is the TMS executive program and synchronization subroutine which provide the capability to simulate many vs many $(\mathrm{MvN})$ aircombat by running multiple, single aircraft simulations in parallel over a network of computer workstations. This paper will describe the three elements of the TMS and provide a demonstration of the operation of the simulation environment.

\section{Description of the Tactical Maneuvering Simulator \\ Aircraft Simulation Model}

Individual aircraft are modeled using a modified version of an existing batch simulation model developed at Langley Research Center. This simulation models a F-18 aircraft with or without a hypothetical, hardware-based thrust-vectoring (TV) system developed by the Northrop Corporation. This TV system uses two vectoring vanes on each engine to provide thrust induced pitch and yaw moments. When necessary to distinguish between the aircraft as equipped with the TV system from the basic aircraft, the basic aircraft will be referred to as the baseline aircraft, while the aircraft with TV will be referred to as the TV aircraft. The batch simulation was developed from the real-time simulation code for the F-18 as implemented in the DMS and from documentation obtained from the McDonnell Aircraft Company (ref 6-9). While an indepth description of the batch simulation is to be published by its authors in a forthcoming NASA report, details relevant to its use in the TMS are presented here.

The computer code implementing the simulation model is written in the Advanced Continuous Simulation Language (ACSL, Reference 10) and FORTRAN. ACSL is a simulation system consisting of a special purpose high-level language, a translator, and various libraries to satisfy the commands available in the language. ACSL simulation models are translated into FORTRAN and linked with the ACSL libraries. The resulting executable program allows interactive user input, and enables the generation of plots and printed outputs. ACSL allows FORTRAN subroutines to be integrated into the simulation model. The simulation uses ACSL to implement the dynamics of the aircraft and engines. Actuator and sensor models are also implemented in ACSL. FORTRAN subroutines are used to calculate aerodynamic forces and moments and steady-state engine parameters. The discrete, inner-loop, control augmentation system of the aircraft is also implemented primarily in FORTRAN.

The equations of motion used in the simulation model the flight of a rigid airplane over a flat, nonrotating Earth using a conventional 6 degree-of -freedom Euler formulation. The mass and moments of inertia of the aircraft are set at the start of a simulation and are assumed to be constant. Typical weights and moments of inertia used for the baseline and thrustvectored aircraft are shown in Table 1. The configuration of the aerodynamic surfaces and controls is shown in Figure 1. The aerodynamic force and moment generated by each surface or control is calculated from a large wind-tunnel-derived database using table look-ups with linear interpolation. Data is stored in nondimensional form as functions of the air data variables [angle-of-attack $(\alpha)$, angle-of-sideslip $(\beta)$, Mach number (M)], the time rate-of-change of $\alpha$ and $\beta$, 
surface deflections, and the body angular rates (p, q, r). The $\alpha$ range is $[-10 \ldots+90]$ degrees, $\beta$ range is [$20 \ldots+20]$ degrees, and $M$ range is [0.2..2.0]. Flexibility effects in the form of flex/rigid ratios and flexibility increments are included in the database to an altitude of 60,000ft. Actuators for all control surfaces except the speedbrake are modeled with a first order lag with time constants and rate limiting, as appropriate. The actuator responsible for moving the speedbrake is modeled as producing a constant deflection rate of 24 degrees per second.

Two engines rated at $16100 \mathrm{lbs}$ of installed static sea level thrust are included in the simulated aircraft. The engine model takes input from the throttle and

current air data [altitude, dynamic pressure $(\overline{\mathrm{q}})$, and $\mathrm{M}$ ] to compute the force currently being produced by the engines. In the case of the TV aircraft, $\alpha$ and $\beta$ effects as well as thrust losses due to vectoring are included in the thrust computation. The TV system consists a two vane per engine installation as shown in Figure 2. By deflecting the thrust of the two engines in a symmetric or anti-symmetric manner, nearly pure pitching or yawing moments can be generated in a manner similar to an aerodynamic $\mathrm{V}$-tail. The actuators for the thrust vectoring vanes are modeled as first order transfer functions with a steady-state gain of one, a time constant of (1/30) seconds, rate limits of 80 degrees per second, and position limits of $\left[ \pm 30^{\circ}\right]$.

The simulated aircraft depends on full authority control augmentation system (CAS) to provide desirable flying qualities throughout its flight envelope. This CAS is documented for the baseline aircraft in detail in References 8 and 9. A simulation of the "Auto Flap Up" mode of the CAS defined by the 8.3.3 production PROM (programmable read only memory) set is included in the simulation model. The CAS used with TV aircraft is a refined and extended version of the baseline CAS. This work was performed by the Flight Dynamics Branch at NASA Langley through extensive batch and piloted simulation analysis. The CAS integrates the TV system with the aerodynamic control surfaces to significantly increase the maneuvering capabilities of the aircraft at high- $\alpha$. The pitch and yaw commands from the command paths of the CAS are divided, as appropriate, between the aerodynamic and TV controls. The pitch and yaw commands sent to the TV system are passed through a mixer which resolves the commands into appropriate vane deflection commands for the thrust-vectoring hardware of the left and right engines.

The CAS augments the dynamics of the bare airframe to provide stability and predictable flying qualities which enable pilots to successfully employ the aircraft in tactical engagements. For use in the TMS, an outer-loop control system is needed around the basic CAS to perform the task of tracking trajectories as commanded by the TDG. In a sense, this outer-loop control system performs the physical functions of the pilot--transforming the desired tactical plan or strategy into actual aircraft motions. This outer-loop control system, known as the Tactical Autopilot, is described in the following section.

\section{Tactical Autopilot}

Most batch air combat simulation environments use simplified aircraft models which only model the steady-state characteristics of an aircraft. Frequently referred to as 5 d.o.f. models, these models are essentially point-mass representations with limitations on the rate at which the aircraft lift vector can be changed in magnitude and orientation. These limitations are selected to reflect the pitch, roll, and yaw capabilities of the simulated aircraft and usually take the form of a set of maximum allowable angular rates. The number of degrees of freedom is 5 rather than 6 because the aircraft is assumed to be coordinated at all times (defined as flight with $\beta=0$ ), requiring $\mathrm{r}=$ ptan $\alpha$. Because no differential equations are used to describe the rotational dynamics of the aircraft, the aircraft orientation can be commanded directly, making the task of executing maneuvers specified by a maneuvering logic trivial.

In contrast, the TMS uses a traditional 6 dof representation of aircraft motion in which both forces and moments are used in the calculation of translational and rotational accelerations. This approach provides an accurate model of transient aircraft motions and is necessary to achieve commonality with piloted simulation models. The difficulty with using this higher-fidelity model is that aircraft attitude can no longer be commanded directly, requiring the addition of an outer-loop autopilot to execute maneuvers commanded by the maneuvering logic. Unlike traditional autopilots, this control system must be able to respond to the large amplitude commands typical of air combat in minimum or near minimum time. In the TMS, the TA (tactical autopilot) has been developed to perform this task.

The function of the TA is to accept trajectory commands generated by the TDG and issue commands to the inner-loop CAS which cause the aircraft to follow the desired trajectory. The TDG issues trajectory commands by specifying a desired $\alpha$ and wind axis bank angle $(\mu)$, defined as 
$\mu=\tan ^{-1}[\quad \mathrm{f}((\sin \theta \cos \alpha \sin \beta+\sin \phi \cos \theta \cos \beta-$ $\cos \phi \cos \theta \sin \alpha \sin \beta),(\sin \theta \sin \alpha+\cos \phi \cos \theta \cos \alpha))]$

combined with a desired throttle and speedbrake setting. Flight with $\beta=0$ is assumed to be desired at all times. For a given flight condition, these parameters determine the magnitude and orientation of the net force vector acting on the aircraft and the attitude or the aircraft relative to an Earth fixed reference system. Since the throttle and speedbrake settings can be obtained directly, no interface is needed to capture these commands; the commands are passed directly from the TDG to the aircraft simulation. The TA thus serves as an allattitude, outer-loop control system to capture and track $\alpha$ and $\mu$ as commanded by the TDG. A block diagram of the complete TDG-TA-aircraft system is shown in Figure 3. It should be recognized that while the TA is described in this report in the context of the TMS, its use is also required in the DMS. By incorporating the TA into the piloted simulation model used in the DMS, the TDG is able to command this simulation in a manner identical to the batch simulation. The design and development of the TA is described in detail in reference 11 and will only be briefly described in this report.

The TA is divided in to two channels; a longitudinal command system which uses longitudinal stick inputs to capture and track commanded $\alpha$, and a lateral command system which uses lateral stick inputs to capture and track the commanded $\mu$. A directional controller is not included in the TA because the innerloop CAS already attempts to maintain zero sideslip, unless commanded not to by using rudder pedal inputs. Piloted simulations have shown that the wind-axis rolling performance performance of the baseline aircraft can be improved slightly at $\alpha$ 's greater than $25^{\circ}$ by combining lateral stick and rudder pedal inputs. This behavior is not being exploited by the current implementation of the TA.

The longitudinal command system uses a proportional-integral-derative (PID) structure with $\alpha$ feedback. The lateral command system uses a proportional-derivative (PD) structure with $\mu$ feedback.

The values of $\alpha, \dot{\alpha}, \mu$, and $\dot{\mu}$ are assumed to be available without error, thus no additional compensation to account for sensor noise or dynamics is included in the TA. There is no attempt to model in the TA the cognitive and neromuscular delays or limitations that would be inherent in a human pilot. Thus, as implemented, the TA represents an idealized controller.

One of the difficulties in developing a system such as the TA is determining suitable criteria to measure the acceptability of the final design. Traditional performance specifications such as frequency and damping are inappropriate considering the largeamplitude, coupled maneuvers performed by the TA. Criteria which reflect the nonlinearities of the task must be used to assess the performance of the TA. The intent of these criteria is to insure that the TA is able to capture and track commands from the TDG in a manner which does not adversely bias the tactical performance of the TDG-TA-aircraft system. Since this tactical performance is dependent on the combined interactions of all three elements, it is desirable to characterize the response of the TA-aircraft system against some functional benchmark. Since the only previous controllers to demonstrate mastery of the simulated aircraft in ACM are human pilots, the performance of pilots performing representative maneuvers should provide a reasonable benchmark for the performance of the TA.

Tables 2 and 3 show the minimum and average time required for a series of experienced pilots perform large amplitude, decoupled $\alpha$ and $\mu$ captures in the baseline and TV aircraft, as simulated in the DMS. Also shown in the tables is the time required by the TA to perform the same captures. All runs start from $1 \mathrm{~g}$, level flight and end when the desired $\alpha$ or $\mu$ is captured within the specified tolerance. The tables show that for all but two of the tasks, the TA required less time than the minimum time used by the pilots. The TA is probably able to consistently perform the desired maneuvers in less time than the human pilots due to its ability to respond instantly to the current situation. In the two tasks in which the TA did not outperform the pilots, the performance differences are small.

For the $90^{\circ}$ roll maneuver at $\alpha=10^{\circ}$ with the TV aircraft, the TA takes 0.06 seconds longer than the minimum piloted time. This increase is probably tactically insignificant and may be due to $\alpha$ variations during the maneuver. Data recorded during the execution of the maneuver show that the pilot allowed $\alpha$ to fall to $7.2^{\circ}$ during the maneuver; the TA experienced a minimum $\alpha$ of $8.5^{\circ}$.

For the $40^{\circ} \alpha$ capture task at Mach 0.6 with the baseline aircraft, the TA was unable to prevent the initial overshoot from exceeding the desired $\pm 2.0^{\circ}$ capture tolerance. This overshoot increased the capture time of the TA for the original capture tolerance beyond the minimum piloted time. The initial overshoot experienced by the TA was $0.44^{\circ}$ beyond the desired capture tolerance. As this overshoot only slightly exceeds the desired capture tolerance, the tactical performance should not be significantly affected. Since attempts to improve the response at this one condition resulted in an overall decrease in system performance, 
the decision was made to accept nominal response of the system. The time listed in Table 3 represents of the performance of the TA with the capture criteria relaxed to $2.44^{\circ}$.

Also shown in the tables is the maximum peak overshoot $\left(\mathrm{M}_{\mathrm{p}}\right)$ for the captures performed by the TA. Burgin, in Reference 12, recommends that for good tactical performance, $\mathrm{Mp}$ for decoupled inputs be limited to $5^{\circ}$ in pitch and $20^{\circ}$ in roll, regardless of the amplitude of the input. For all the captures, the TA is below these recommended limits.

The capture tasks shown above measure performance for single-axis, step-inputs. In ACM the TA will be expected to respond to sequences of simultaneous $\alpha$ and $\mu$ commands. The response of the TA to a representative command sequence is shown in Figures 4 and 5 for the baseline and TV aircraft, respectively. These command sequences were obtained by discretizing, at one second intervals, continuous $\alpha$ and $\mu$ time histories recorded during piloted ACM engagements. This discretization was performed to obtain command sequences representative of the command update rate of the TDG. Because the sequences were obtained from actual trajectories, they should be reasonably close to the capabilities of the TA controlled aircraft and representative of a tactically realistic sequence.

The TA appears to follow both sequences with sufficient accuracy to effectively implement realistic maneuver sequences. As shown in Figures 4 and 5, the ability of the TA to capture and maintain $\alpha$ and $\mu$ is only slightly reduced by the coupled command sequences. It should be noted that an absolute, operational assessment of the effectiveness of the TA cannot be performed until the system is interfaced with an appropriate TDG and tested against human pilots in the DMS.

\section{Multiple Aircraft Simulation}

In contrast to most batch simulation environments which are implemented as a single large process, the TMS uses a concurrent, parallel implementation structure to provide multi-aircraft simulation. This parallel implementation allows a single copy of the simulation program to be run concurrently as needed to simulate the individual engagement participants. The number of concurrent copies of the simulation which can be executed simultaneously is only limited by available computer memory and the desired execution speed--of course, an appropriate TDG would be needed to command this number of aircraft.

Parallel implementation offers several other key advantages over conventional methods. Since all aircraft are simulated by the same program, corrections or updates to this model need only be performed once, easing configuration control. The parallel implementation also allows different simulation models to be incorporated into the TMS and intermixed with the current aircraft simulation model with the addition of a standard subroutine. Thus, simulations of different aircraft types can easily be added to the TMS, allowing comparisons of the tactical performance of dissimilar aircraft. Simulations which may be added to the TMS are not restricted to aircraft; high-fidelity missile simulations could also be implemented in a similar fashion. Finally, parallel implementation allows individual simulations to be distributed on multiple, networked computers, reducing the time required to simulate a given engagement.

While the concurrent parallel implementation provides the above mentioned benefits, it is necessary to provide a control mechanism to synchronize the otherwise independently executing simulations. This synchronization is required so that the simulations remain together on the same time step. Since the simulations execute as independent processes on a given computer (or set of computers), the order and length of time in which the computer operates on each process is a function of other jobs which may be executing on the machine and is essentially indeterminate. Thus without some control mechanism, the simulations may progress at different rates.

The TMS utilizes a read-write synchronization protocol to suspend execution of individual processes at a specified point until all relevant processes have reached this point. The protocol is used in the TMS to suspend execution of the aircraft simulations at the end of the current time step or simulation frame. The simulations are allowed to proceed only after all the participating simulations have reached the end of the current time step and have received updated maneuvering commands from their controlling TDG.

The key elements of the parallel implementation are an executive program, a communication and synchronization subroutine called by the aircraft simulation model, and a specialized message-passing protocol. The executive program serves as a master process which initializes the individual simulation models and supervises their operation in a common reference frame. The executive program also manages communications with the TDG. Since all communication between a TDG and its corresponding aircraft must pass through the executive, the flow of information can be closely monitored and controlled. The communication and synchronization subroutine is called by the aircraft simulation model at the completion of each simulation frame. By means of the message-passing protocol, this subroutine allows the 
executive program to suspend execution of the simulations, pass current state information from the simulations to the maneuvering logics, and return updated maneuver commands to the simulations at the end of the decision process.

The following section demonstrates the capabilities of the TMS with a sample engagement.

\section{Demonstration of The Tactical Maneuvering Simulator}

This example engagement demonstrates a $1 \mathrm{v} 1$ dogfight between a drone aircraft following a predefined, open-loop command sequence and an aircraft actively guided by a simple TDG. The objective of this example is to demonstrate the operation of the TMS with an active TDG.

The TDG commands $\alpha$ and $\mu$ in an effort to cause the flight path of the guided aircraft to intersect a predicted future position of the drone aircraft. This predicted future position is obtained by extrapolating along a second order curve fit to the past three recorded positions of the drone aircraft. The TDG then determines the maneuverplane and load factor required to intercept this position given the current state of the guided aircraft. The required maneuverplane and load factor are converted into a required $\alpha$ and $\mu$. If the required load factor is outside the aerodynamic or structural capabilities of the aircraft, $\alpha$ corresponding to maximum available or allowable lift is commanded. In addition, if the commanded $\mu$ differs from the current $\mu$ by more than $45^{\circ}$ and the commanded $\alpha$ is greater than $15^{\circ}$, the $\alpha$ command is reduced to $15^{\circ}$ in order to expedite the execution of the rolling maneuver. This $\alpha$ reduction was heuristically selected and does not necessarily reflect an optimum maneuvering strategy.

The engagement between the two aircraft is shown in Figure 6 from various perspectives. The engagement starts with both aircraft trimmed in $1 \mathrm{~g}$ level flight at an altitude of 10,000ft and Mach=0.9. The aircraft start from opposite headings with a $10,000 \mathrm{ft}$ longitudinal separation and a 1,000ft lateral offset. The drone aircraft is initially commanded to maintain $\mu=0^{\circ}$ and increase $\alpha$ slightly over the trim value. The throttle of the drone aircraft is advanced into the afterburner region. These commands are maintained during the first 10 seconds of the engagement. After the initial merge, the guided aircraft performs an oblique, pitch-back maneuver to reverse its heading back toward the drone aircraft. Following this initial 10 second period, the drone is commanded to increase $\alpha$ to $28^{\circ}$ and alternate $\mu$ between $\pm 90^{\circ}$, switching every 10 seconds. The resulting motion is a descending spiral like trajectory. In response to these maneuvers, the guided aircraft reverses its heading again, and effectively tracks the drone down the descending spiral. Time histories comparing commanded $\alpha$ to actual $\alpha$ and commanded $\mu$ to actual $\mu$ for the guided aircraft are shown in Figure 7. These time histories demonstrate that the TA controlled aircraft is able to closely track the guidance commands generated by the TDG.

\section{Current Research Activities}

The TMS, as part of TiGRES, is being used to investigate and develop tactics for highly agile aircraft. The tactical capability of the TV equipped aircraft is to be compared with the baseline aircraft in 1 versus 1 and 1 versus 2 scenarios. This comparison requires the development of a TDG capable of maneuvering the aircraft effectively in these scenarios. A prototype TDG known as the Computerized Logic for Air Warfare Simulation (CLAWS) has been developed for 1 versus 1 air combat using simplified 5 dof aircraft models (Ref 4). An extension of CLAWS, known as Paladin, has been interfaced with the TMS and is currently being evaluated with the high-fidelity aircraft models used in the TMS (Ref 13).

The TA as described in this paper only supports guidance commands in the form of a desired $\alpha$ and $\mu$. These parameters are useful for commanding the trajectory of an aircraft during the gross maneuvering phases of air combat maneuvering. However, when a target has been acquired, and fine tracking is required to achieve a weapons solution, having a direct means of "aiming" the aircraft is desirable. To support this desire, a second mode of operation is being added to the TA. This mode will allow the TDG to designate a target to the TA and the TA will then use a conventional feedback control law to minimize the lineof-sight error to the target.

\section{Concluding Remarks}

The development and operation of a batch air combat simulation environment known as the Tactical Maneuvering Simulator has been presented. The Tactical Maneuvering Simulator serves as a tool for developing and evaluating tactical maneuvering logics. The environment can also be used to evaluate the tactical implications of perturbations to aircraft performance and supporting systems.

The Tactical Maneuvering Simulator was developed using an existing batch simulation of a modern high-performance aircraft, with and without thrust-vectoring. This batch simulation uses 6 degreeof-freedom equations of motion, aerodynamics, propulsive characteristics, and control laws equivalent to those used in high-fidelity piloted simulation.

An outer-loop control system known as the Tactical Autopilot was developed to allow maneuvering 
logics to command the 6 degree-of-freedom aircraft model. The Tactical Autopilot uses longitudinal and lateral stick inputs to capture angle-of-attack and windaxis bank angle as commanded by the maneuvering logic. The performance of the Tactical Autopilot was demonstrated by comparing the time required for it to capture decoupled angle-of-attack and bank-angle commands to the time required by human pilots for the same commands. The performance of the the Tactical Autopilot was equivalent or superior to the pilots for nearly all the commands investigated. The ability of the Tactical Autopilot to track realistic command sequences of angle-of-attack and bank-angle was demonstrated using sequences generated from piloted air combat simulations. The Tactical Autopilot was shown to effectively track these representative command sequences.

To provide for the simulation of air combat with multiple participants, a parallel implementation scheme was developed using a read-write synchronization protocol. This parallel implementation allows the Tactical Maneuvering Simulator to simulate air combat with any number of engagement participants. The maximum number of participants is limited only by the available computer resources. The parallel implementation is also beneficial from the standpoint of simplifying software maintenance and allowing new simulations to be easily added to the environment.

The capabilities of the Tactical Maneuvering Simulator were demonstrated with an example engagement. This engagement demonstrated the ability of the environment to simulate multiple aircraft and to interact with an active tactical decision generator. The tactical autopilot was shown to closely follow the maneuver commands from the tactical decision generator.

\section{References}

1. Advanced Air-to-Air System Performance Model (ASSPEM) Users Manual, Boeing Document D180-28938-1, November 1985.

2. Kerchner, R. M.; et al.: The TAC BRAWLER Air Combat Simulation Analyst Manual (Rev. 3.0). Decision Science Applications Report \#668, 1985 .

3. Goodrich, K. H.; and McManus, J. W.: Development of a Tactical Guidance Research and

Table 1 - Summary of Weight, CG and Inertias
Evaluation System (TiGRES). AIAA Paper 893312, August 1989.

4. McManus, J. W.; and Goodrich, K. H.: Application of Artificial Intelligence (AI) Programming Techniques To Tactical Guidance for Fighter Aircraft. AIAA Paper 89-3525, August 1989.

5. Ashworth, B. R.; and Kahlbaum, William M., Jr: Description and Performance of the Langley Differential Maneuvering Simulator. NASA TN D-7304, 1973.

6. F/A-18 Stability and Control Data Report, Vol I: Low Angle of Attack. McDonnell Aircraft Company, MDC A7247, November 1982.

7. F/A-18 Stability and Control Data Report, Vol II: High Angle of Attack. McDonnell Aircraft Company, MDC A7247, August 1981.

8. F/A-18 Flight Control Electronic Set Control Laws, Volume I. McDonnell Aircraft Company, MDC A4107, July 1988.

9. F/A-18A Flight Control System Design Report, Volume I, System Description and Theory of Operation. McDonnell Aircraft Company, MDC A7813, September 1984.

10. Advanced Continuous Simulation Language (ACSL) Reference Manual, Fourth Edition. Mitchell and Gauthier Associates, 1986.

11. Goodrich, K.H.: Development of a Six Degreeof-Freedom Simulation Environment for Tactical Guidance Research and Evaluation. Masters Thesis, George Washington University, April 1991

12. Burgin, G. H.; Eggleston D. M.: Design of an All-Attitude Flight Control System to Execute Commanded Bank Angles and Angles of Attack. NASA CR-145004, February 1976.

13. Chappell, A. R.; McManus J. W.; Goodrich, K. H.: Trail Maneuver Generation and Selection In the Paladin Tactical Decision Generation System. AIAA Paper 92-4541, August 1992 
CG Locations

Weight

Fuselage

Station

(in)

Thrust-Vectored

Baseline (lbs)

33310

31665
455.0

457.3
Water

Line

Moments and Products of Inertia

$\begin{array}{lll}\mathrm{I}_{\mathrm{yy}} & \mathrm{I}_{\mathrm{zz}} & \mathrm{I}_{\mathrm{Xz}}\end{array}$

$\left(\right.$ slug $\left.-\mathrm{ft}^{2}\right)$

\begin{tabular}{lllll}
02.8 & 23000 & 151293 & 169945 & -2971 \\
01.6 & 22337 & 120293 & 138945 & -2430 \\
\hline
\end{tabular}

Table 2 - Time Required by TA to Perform $\alpha$ Captures

All runs started at altitude $=25,000 \mathrm{ft}$

\begin{tabular}{|c|c|c|c|c|c|c|c|c|}
\hline Aircraft & $\begin{array}{c}\text { Initial } \\
\alpha \\
\text { (deg) }\end{array}$ & $\begin{array}{c}\text { Final } \\
\alpha \\
(\mathrm{deg})\end{array}$ & $\begin{array}{l}\text { Initial } \\
\text { Mach }\end{array}$ & $\begin{array}{l}\text { Capture } \\
\text { Criteria }\end{array}$ & $\begin{array}{c}\text { Average } \\
\text { Time by } \\
\text { Pilot } \\
(\mathrm{sec})\end{array}$ & $\begin{array}{c}\text { Minimum } \\
\text { Time by } \\
\text { Pilot } \\
(\mathrm{sec}) \\
\end{array}$ & $\begin{array}{c}\text { Time by } \\
\text { TA } \\
(\mathrm{sec})\end{array}$ & $\begin{array}{l}\text { Maximum } \\
\text { Overshoot } \\
\text { (deg) }\end{array}$ \\
\hline Baseline & 4.4 & 30.0 & 0.60 & $+/-2^{\circ}$ & 5.12 & 4.35 & 1.91 & 1.9 \\
\hline " & 4.4 & 40.0 & 0.60 & $"$ & 2.88 & 2.30 & $2.28^{*}$ & 2.4 \\
\hline " & 23.5 & 30.0 & 0.30 & $"$ & 4.93 & 3.78 & 1.00 & 1.4 \\
\hline$"$ & 23.5 & 40.0 & 0.30 & $"$ & 6.56 & 5.95 & 1.81 & 1.6 \\
\hline$"$ & 10.0 & 0.0 & 0.40 & $"$ & 2.50 & 1.99 & 1.34 & 1.0 \\
\hline$"$ & 20.0 & 0.0 & 0.32 & $"$ & 5.86 & 5.25 & 1.88 & 2.0 \\
\hline$"$ & 30.0 & 0.0 & 0.27 & $"$ & 7.06 & 5.68 & 2.38 & 2.0 \\
\hline TV & 4.4 & 30.0 & 0.60 & $"$ & 4.70 & 3.84 & 1.09 & 1.7 \\
\hline$"$ & 4.4 & 40.0 & 0.60 & $"$ & 4.45 & 3.46 & 2.97 & 2.6 \\
\hline$"$ & 4.4 & 50.0 & 0.60 & $"$ & 4.76 & 5.31 & 2.41 & 0.2 \\
\hline$"$ & 23.5 & 30.0 & 0.30 & $"$ & 2.11 & 1.09 & 0.81 & 1.2 \\
\hline$"$ & 23.5 & 40.0 & 0.30 & $"$ & 2.69 & 1.41 & 1.38 & 1.2 \\
\hline$"$ & 23.5 & 50.0 & 0.30 & $"$ & 3.39 & 1.79 & 1.78 & 1.6 \\
\hline$"$ & 10.0 & 0.0 & 0.40 & $"$ & 2.18 & 2.18 & 1.12 & 0.4 \\
\hline$"$ & 20.0 & 0.0 & 0.32 & $"$ & 2.11 & 1.66 & 1.60 & 0.7 \\
\hline$"$ & 30.0 & 0.0 & 0.27 & $"$ & 4.60 & 4.54 & 1.89 & 0.6 \\
\hline
\end{tabular}

* Capture criteria relaxed to $2.4^{\circ}$

7

8

9

10

11

12

13

Table 3 - Time Required by TA to Perform $90^{\circ} \mu$ Captures

All runs started at altitude $=25,000 \mathrm{ft}$

\begin{tabular}{|c|c|c|c|c|c|c|c|c|}
\hline Aircraft & $\begin{array}{c}\text { Initial } \\
\alpha \\
(\mathrm{deg})\end{array}$ & $\begin{array}{c}\text { Initial } \\
\mu \\
(\mathrm{deg})\end{array}$ & $\begin{array}{c}\text { Final } \\
\mu \\
(\mathrm{deg})\end{array}$ & $\begin{array}{c}\text { Capture } \\
\text { Criteria }\end{array}$ & $\begin{array}{c}\text { Average } \\
\text { Time by } \\
\text { Pilot } \\
(\mathrm{sec})\end{array}$ & $\begin{array}{c}\text { Minimum } \\
\text { Time by } \\
\text { Pilot } \\
(\mathrm{sec})\end{array}$ & $\begin{array}{c}\text { Time by } \\
\text { T A } \\
(\mathrm{sec})\end{array}$ & $\begin{array}{c}\text { Maximum } \\
\text { Overshoot } \\
(\mathrm{deg})\end{array}$ \\
\hline Baseline & 10.0 & 0.0 & 90.0 & $+/-5^{\circ}$ & 4.10 & 3.07 & $\mathbf{1 . 4 3}$ & $\mathbf{3 . 8}$ \\
\hline$"$ & 20.0 & 0.0 & 90.0 & $+/-8^{\circ}$ & 8.90 & 6.70 & $\mathbf{4 . 9 0}$ & $\mathbf{6 . 0 0}$ \\
\hline TV & 10.0 & 0.0 & 90.0 & $+/-5^{\circ}$ & 2.15 & 1.47 & $\mathbf{1 . 5 3}$ & $\mathbf{2 . 8}$ \\
\hline$"$ & 20.0 & 0.0 & 90.0 & $+/-5^{\circ}$ & 5.00 & 4.40 & $\mathbf{2 . 2 2}$ & $\mathbf{2 . 7}$ \\
\hline$"$ & 30.0 & 0.0 & 90.0 & $+/-5^{\circ}$ & 5.17 & 2.75 & $\mathbf{2 . 5 0}$ & $\mathbf{3 . 9}$ \\
\hline
\end{tabular}

